\title{
MEASURABLE APPROXIMATION OF A SECOND-ORDER PROCESS
}

\author{
JELENA B. GILL
}

\begin{abstract}
An important problem, having practical as well as theoretical significance, is the problem of determining whether or not a stochastic process has a measurable modification or can be approximated (in the mean square sense) by a second-order process admitting such a modification. It is known [1] that a secondorder process has a measurable modification if and only if its covariance function is measurable and its linear space is separable. However, a problem still open is whether sufficient conditions for the existence of a measurable modification can be given in terms of the covariance function only, so that they have a pure analytical form. In this paper we formulate one set of such conditions (Theorem 2, Corollary 2.2) and from that derive a result giving conditions under which a second-order process can be uniformly approximated (in the mean square sense) by a measurable second-order process (Theorem 3).
\end{abstract}

Introduction. Let $X=X(t), t \in(0 ; 1)$, be a real-valued second-order stochastic process and let $R=R(t, u)=E[X(t) X(u)], t, u \in(0 ; 1)$, be its covariance function. Let $(\Omega, \mathscr{F}, P)$ be a probability space on which all random variables under consideration are defined. The linear space of $X$ will be denoted by $H(X)$ : $H(X)=\overline{\mathscr{L}}\{X(t), t \in(0,1)\} ; H(X)$ is a Hilbert space and $R(t, u)$ is an inner product between $X(t)$ and $X(u)$. A process $X$ is said to be measurable if it is measurable with respect to $\mathscr{F} \times \mathscr{B}$, where $\mathscr{B}$ is a Borel $\sigma$-field over $(0 ; 1)$. A process $Y=Y(t), t \in(0 ; 1)$, is said to be a modification of $X$ if $P\{X(t)=Y(T)\}=1$ for each $t \in(0 ; 1)$.

It is known [1] that a second-order process $X$ has a measurable modification if and only if its covariance function is measurable and its linear space is separable. However, it is not clear what latitude can be allowed in the behavior of the covariance function of a stochastic process admitting a measurable modification. Obviously, a continuous covariance function corresponds to a process with a measurable modification, but it would be useful to determine how much this continuity can be weakened without altering the latter property. In this paper we are looking for conditions expressed in terms of approximate continuity. The motivation for this approach is the following. Suppose that a mean square continuous process $X$ (which has a measurable modification) is "disturbed" by some "noise." The question

Received by the editors May 18, 1984 and, in revised form, September 25, 1985 and March 3, 1986. 1980 Mathematics Subject Classification (1985 Revision). Primary 60G17, 60G07.

Key words and phrases. Second-order stochastic process, approximate continuity, covariance function, measurable modification, mean square oscillation, orthogonal dimension. 
is: how much (in the mean square sense) can $X$ be disturbed and still maintain a measurable modification? More precisely: how weak a property can take the place of the mean square continuity of $X$ without affecting the existence of its measurable modification? In approaching this question, the following two things are to be kept in mind: (1) sufficient conditions should be given in terms of covariance function, and (2) measurability of a real-valued function is equivalent to its approximate continuity almost everywhere [5, Theorem 42.3]. That is why we prefer to give the following form to the above question: can sufficient conditions for the existence of a measurable modification of $X$ be formulated in terms of approximate continuity of its covariance function? Since Davies' result from [2, Theorem 1] implies that, if all functions from $\mathscr{R}=\left\{R_{t}(\cdot)=R(t, \cdot), t \in(0 ; 1)\right\}$ are approximately continuous, then function $R$ is measurable (as a function of two variables), it appears that there is sense in investigating what impact the approximate continuity of all functions from $\mathscr{R}$ will have on the separability of the linear space of $X$ (and thus [1] on the measurability of $X$ as well). In this paper, we shall investigate that impact (Theorem 2 and Corollary 2.2) and, by using some obtained results, derive conditions under which a second-order process can be uniformly approximated almost everywhere on a set of arbitrarily small Lebesgue measure by a measurable second-order process with continuous trajectories (Theorem 3 ).

Definitions and results. Let $m$ be a Lebesgue measure on $(0 ; 1)$ and let $A$ be any set from $(0 ; 1)$. It is said that $A$ has metric density $\lambda$ at $u \in(0 ; 1)$ if

$$
m(A \cap(u-\varepsilon ; u+\varepsilon)) / 2 \varepsilon
$$

converges to $\lambda$ when $\varepsilon>0$ converges to zero. A real-valued function $f$, defined on $(0 ; 1)$, is said to be approximately continuous at $u \in(0 ; 1)$ if there is a set $A_{u}$, having the unit metric density at $u$, along which $f$ is continuous at $u[4,5] ; f$ is approximately continuous on $A$ if it is approximately continuous at each point from $A$. A continuous function is obviously approximately continuous, but the converse does not hold. For instance, if $f$ is equal to zero at all rational points and equal to some continuous function at all irrational points, then $f$ is approximately continuous at each irrational point, although it is discontinuous everywhere. A necessary and sufficient condition for a function to be measurable is that it is approximately continuous almost everywhere [5, Lemma 42.3].

Mean square approximate continuity of a stochastic process is defined in a way analogous to that one for a real-valued function and the only difference is that convergence of a sequence of real numbers is replaced by mean square convergence of random variables.

For a given process $X$, let us consider function $R_{t}=R_{t}(\cdot)=E[X(t) X(\cdot)]$ for some fixed $t \in(0 ; 1)$. For some $u \in(0 ; 1)$ and $\varepsilon>0$, set $B_{t, u ; \varepsilon}$ will be defined by $B_{t, u ; \varepsilon}=\left\{v:\left|R_{t}(u)-R_{t}(v)\right| \leqslant \varepsilon\right\}$. If $R_{t}$ is approximately continuous at $u$, then $B_{t, u ; \varepsilon}$ has unit metric density at $u$ for each $\varepsilon>0$. We shall say that functions from $\mathscr{R}=\left\{R_{t}, t \in(0,1)\right\}$ are uniformly approximately continuous with respect to $t$ if, for all $t, u \in(0 ; 1)$ and $\varepsilon>0$, sets $B_{t, u ; \varepsilon}$ depend only on $u$ and $\varepsilon$ and not on $t$; if this is the case we shall, for any $t$, write $B_{u, \varepsilon}$ instead of $B_{t, u ; \varepsilon}$. 
The following fact (see [4, p. 194] and [5, Lemma 42.1]) will be repeatedly used in the sequel: If $S$ is a set of positive Lebesgue measure, then there is a set $S^{*} \subseteq S$, $m\left(S^{*}\right)=m(S)$, which has the unit metric density at each of its points.

Now, let us return to the problem of determining conditions under which process $X$ has a measurable modification or can be approximated (in the mean square sense) by such processes. Let function $g$ be defined by $g(t)=R(t, t), t \in(0 ; 1)$, where $R$ is covariance function of $X$.

THEOREM 1. If functions from $\mathscr{R}$ are uniformly approximately continuous with respect to $t$, then function $g$ is measurable.

Proof. The assumption that $g$ is not measurable is equivalent to the existence of a set $S$ of positive Lebesgue measure on which $g$ is not approximately continuous. Let $S^{*}, S^{*} \subseteq S, m\left(S^{*}\right)=m(S)$, be a set of unit metric density at each of its points. Since $g$ is not approximately continuous at points from $S^{*}$, it means that for each $t \in S^{*}$ there are $\varepsilon_{t}>0$ and a set $S_{t ; \varepsilon_{t}}$ of positive metric density at $t$, such that

$$
|g(t)-g(u)|>\varepsilon_{t}, \quad u \in S_{t ; \varepsilon_{t}} .
$$

It is clear that set $S_{t ; \varepsilon_{t}}$ can be chosen so that $S_{t ; \varepsilon_{t}} \subseteq S^{*}$.

Let us show the following: for each $t \in S^{*}$ there is $\delta>0$ such that a set $A_{t ; \delta}$ of points $u \in S^{*}$, having sets $S_{u ; \delta}$ (of positive metric density at $u$ ) with the property

$$
|g(u)-g(v)|>\delta, \quad v \in S_{u ; \delta},
$$

has unit metric density at $t$. Let $t \in S^{*}$ be arbitrary but fixed and let $\delta_{1}, \delta_{2}, \ldots>0$ be a sequence decreasing to zero. If the metric density at $t$ of each set $A_{t ; \delta_{i}}$ is smaller than one, then (because $A_{t ; \delta_{i}} \subseteq A_{t ; \delta_{j}}$ for $i \leqslant j$ ) the metric density at $t$ of set $\bigcup_{i=1}^{\infty} A_{t ; \delta_{i}}$ must also be smaller than one, which contradicts the fact that $S^{*}$ has the unit metric density at each of its points. Thus, there is $\delta=\delta_{t}$ for which $A_{t ; \delta_{t}}$ has the unit metric density at $t$. It is clear that this $\delta_{t}$ and $\varepsilon_{t}$ from (1) can be chosen so that $\delta_{t}=\varepsilon_{t}$. In the sequel it will be assumed that this is done.

From the assumption that functions from $\mathscr{R}$ are uniformly approximately continuous with respect to $t$ it follows that, for each $\varepsilon>0$, there is a set $B_{t ; \varepsilon}$ of unit metric density at $t$, such that $\left|R_{t}(t)-R_{t}(u)\right| \leqslant \varepsilon, u \in B_{t ; \varepsilon}$. It is clear that set $B_{t ; \varepsilon} \cap A_{t ; \delta_{t}}$ has unit metric density at $t$ for each $\varepsilon>0$. Since set $S_{t ; \delta_{t}}$ has positive metric density at $t$, it can be chosen so that $S_{t ; \delta_{t}} \subseteq B_{t: \delta_{t} / 4} \cap A_{t ; \delta_{t}}$.

Let $S_{t ; \delta_{t}}^{*}, S_{t ; \delta_{t}}^{*} \subseteq S_{t ; \delta_{t}}, m\left(S_{t ; \delta_{t}}^{*}\right)=m\left(S_{t ; \delta_{t}}\right)$, be a set of unit metric density at each of its points and let $u$ be an arbitrary point from $S_{t: \delta_{t}}^{*}$. Since $S_{t: \delta_{t}}^{*} \subseteq S_{t ; \delta_{t}} \subseteq B_{t: \delta_{t} / 4} \cap$ $A_{t ; \delta_{t}}$, it means that there is a set $S_{u ; \delta_{t}}$ of positive metric density at $u$, such that (because $u \in A_{t ; \delta_{t}}$ means that $\delta_{u}=\delta_{t}$ )

$$
|g(u)-g(v)|>\delta_{t}, \quad v \in S_{u: \delta_{t}} .
$$

Set $S_{u: \delta_{t}}$ can be chosen so that $S_{u ; \delta_{t}} \subseteq S_{t ; \delta_{t}}^{*}$ (because $S_{t ; \delta_{t}}^{*}$ has unit metric density at each of its points), which implies

$$
\left|R_{t}(v)-R_{t}(u)\right| \leqslant\left|R_{t}(t)-R_{t}(v)\right|+\left|R_{t}(t)-R_{t}(u)\right| \leqslant \frac{1}{2} \delta_{t}, \quad v \in S_{u ; \delta_{t}} .
$$


The assumption that all functions from $\mathscr{R}$ are uniformly approximately continuous with respect to $t$, and (3) imply

$$
\left|R_{v}(v)-R_{v}(u)\right| \leqslant \frac{1}{2} \delta_{t}, \quad v \in S_{u ; \delta_{t}}
$$

Finally, from (2) and (4) one gets

$$
\delta_{t}<|g(u)-g(v)| \leqslant \frac{1}{2} \delta_{t}+\left|R_{u}(v)-R_{u}(u)\right|, \quad v \in S_{u ; \delta_{t}},
$$

that is,

$$
\left|R_{u}(v)-R_{u}(u)\right|>\frac{1}{2} \delta_{t} \quad \text { for each } v \in S_{u ; \delta_{t}}
$$

Since $S_{u ; \delta_{t}}$ has positive metric density at $u$, the last inequality contradicts the assumption that $R_{u}$ is approximately continuous at $u$. The proof is completed.

COROLlARY 1.1. If all functions from $\mathscr{R}$ are uniformly approximately continuous with respect to $t$, then $X$ is mean square approximately continuous almost everywhere.

Proof follows form Theorem 1 and inequality

$$
E|X(t)-X(u)|^{2} \leqslant 2\left|R_{t}(t)-R_{t}(u)\right|+|g(t)-g(u)| .
$$

The following result gives necessary conditions for a stochastic process to have a measurable modification almost everywhere, while its second corollary solves one of the problems formulated at the beginning of this paper.

THEOREM 2. If the conditions

$\left(\mathrm{C}_{1}\right)$ all functions from $\mathscr{R}$ are uniformly approximately continuous with respect to $t$, and

$\left(\mathrm{C}_{2}\right)$ each function $R_{t}$ is continuous from at least one side at $t$,

are satisfied, then there is a measurable process $X_{m}$ such that set $\left\{t: P\left\{X_{m}(t) \neq\right.\right.$ $X(t)\}>0\}$ has m-measure zero.

Proof. This theorem will be proved if we show that, for each $\varepsilon>0$, there is a closed set $E_{\varepsilon}$ such that $m\left(E_{\varepsilon}^{c}\right)<\varepsilon$ and that the linear space $\overline{\mathscr{L}}\left\{X(t), t \in E_{\varepsilon}\right\}$ is separable. Namely, in that case, for example, process $X_{\varepsilon}(t)=I_{E_{\varepsilon}}(t) X(t), t \in(0 ; 1)$, will be measurable (see [2, Theorem 1] and [1, Theorem 1]), which will mean, because $\varepsilon>0$ is arbitrary, that $X$ is almost everywhere equal to a measurable process, as we wanted to prove.

It is easy to see that process $X$ is mean square continuous from at least one side everywhere on a set of arbitrary small measure. Indeed, since function $g$ is measurable (according to Theorem 1), the Lusin Theorem (see e.g. [5, Theorem 21.4]) implies that, for each $\varepsilon>0$, there is a closed set $F_{\varepsilon}, m\left(F_{\varepsilon}^{c}\right)<\varepsilon$, such that $g$ is continuous at every point from $F_{\varepsilon}$, which (together with (5) and $\left(\mathrm{C}_{2}\right)$ ) means that $X$ is mean square continuous from at least one side at every point from $F_{\varepsilon}$.

Let us show that, for each $\varepsilon>0$, set $F_{\varepsilon}$ contains only at most countably many values $t$ such that $X(t-0)$ does not exist $\left(X(t-0)\right.$ is defined as 1.i.m. ${ }_{u \rightarrow t-0} X(u)$, provided this limit exists). Suppose that this is not true, that is, for some $\varepsilon>0$, set 
$F_{\varepsilon}$ contains continuously many values $t$ for which $x(t-0)$ does not exist. Let $\sigma(t)$ be a value of the mean square oscillation function of $X$ at $t$ :

$$
\sigma(t)=\operatorname{Inf}_{\varepsilon \rightarrow 0} \operatorname{Sup}_{t ; t^{\prime \prime} \varepsilon(t-\varepsilon ; t+\varepsilon)}\left\|X\left(t^{\prime}\right)-X\left(t^{\prime \prime}\right)\right\|, \quad t \in(0 ; 1) .
$$

Obviously, there is $\sigma>0$ such that the inequality $\sigma(t)>\sigma$ holds for continuously many values $t \in F_{\varepsilon}$. Furthermore, there is $t^{*} \in F_{\varepsilon}$ such that $\sigma\left(t^{*}\right)>\sigma$ and such that each right neighborhood of $t^{*}$ contains infinitely many values $t \in F_{\varepsilon}$ such that $\sigma(t)>\sigma$ (if every $t \in F_{\varepsilon}$, for which $\sigma(t)>\sigma$, has a right neighborhood with only finitely many values $u \in F_{\varepsilon}$ such that $\sigma(u)>\sigma$, then $F_{\varepsilon}$ itself contains only at most countably many values $t$ satisfying $\sigma(t)>\sigma$, contrary to our hypothesis). Since $t^{*} \in F_{\varepsilon}, X\left(t^{*}+0\right)$ exists and $X\left(t^{*}+0\right)=X\left(t^{*}\right)$. Hence, for a small enough right neighborhood 0 of $t^{*}$,

$$
\left\|X\left(t^{*}\right)-X(t)\right\|<\sigma / 4, \quad t \in o .
$$

Let $t \in o$ be such that $\sigma(t)>\sigma$. For arbitrary $t^{\prime}, t^{\prime \prime} \in\left(t^{*} ; t\right)$ it will be (because of the previous inequality) $\left\|X\left(t^{\prime}\right)-X\left(t^{\prime \prime}\right)\right\|<\sigma / 2$, which will give $\sigma(t)<\sigma / 2$, contrary to the assumption $\sigma(t)>\sigma$. In this way it is proved that $F_{\varepsilon}$ contains only at most countably many values $t$ such that $X(t-0)$ does not exist.

If it is shown that

$$
\operatorname{dim} \overline{\mathscr{L}}\left\{X(t), t \in F_{\varepsilon}\right\} \leqslant \aleph_{0}
$$

(dim denotes the orthogonal dimension), that will mean that sets $F_{\varepsilon}$ are exactly those sets $E_{\varepsilon}$ we were looking for, so that the proof will be completed. A proof for (6) is based on the following fact. If $\left\{x_{\nu}\right\}$ is an orthogonal basis of the linear space $\overline{\mathscr{L}}\left\{X(t), t \in F_{\varepsilon}\right\}$ and if $t \in F_{\varepsilon}$ is such that $X(t-0)$ exists, then the following is true: if, for some $\nu, X(t-0)$ is not orthogonal to $x_{\nu}$, then in each left neighborhood of $t$ there is at least one rational number $r_{t}$ such that $X\left(r_{t}\right)$ is not orthogonal to $x_{v}$. Indeed, if $X(t-0)$ is not orthogonal to $x_{\nu}$, but $X(r)$ is orthogonal to $x_{\nu}$ for each rational number $r<t$ from some left neighborhood $n$ of $t$, then

$$
\|X(t-0)-X(r)\|>\left\|P_{\overline{\mathscr{L}}\left\{x_{\nu}\right\}} X(t-0)\right\|, \quad r \in n(r \text { rational })
$$

( $P$. is a projection operator on a space in the subscript), which contradicts the assumption that $X(t-0)$ is a unique left mean square limit of $X$ at $t$. Thus, if $X(t-0), t \in F_{\varepsilon}$, exists, then all elements from $\left\{x_{\nu}\right\}$ taking place in the orthogonal decomposition of $X(t-0)$ must take place in the orthogonal decomposition of at least one $X(r)$ ( $r$ is a rational number, not necessarily from $F_{\varepsilon}$ ) in each left neighborhood of $t$. Let $S$ be a set containing all rational numbers from $(0 ; 1)$, all points $t \in F_{\varepsilon}$ for which $X(t-0)$ exists but is not equal to $X(t)$, and all points $t \in F_{\varepsilon}$ for which $X(t-0)$ does not exist. Set $S$ is countable (for, if there are continuously many values $t \in S$ such that $X(t-0)$ exists but is not equal to $X(t)$, that will mean that $X(t-0)$ does not exist for continuously many values of $t$ (see [4, p. 130]), contrary to the above conclusion) and thus $\operatorname{dim} \overline{\mathscr{L}}\{X(t), t \in S\} \leqslant \boldsymbol{\aleph}_{0}$. Inequality (6) will be proved if it is shown that

$$
\operatorname{dim} \overline{\mathscr{L}}\left\{X(t), t \in F_{\varepsilon}\right\} \leqslant \operatorname{dim} \overline{\mathscr{L}}\{X(t), t \in S\} .
$$


To prove this, let us suppose that arbitrary $x_{\nu}$ from $\left\{x_{\nu}\right\}$ takes part in the representation of $X(t)$ for some $t \in F_{\varepsilon}$. If $t \in S$, then $x_{\nu}$ belongs to the linear space on the right side of (7). If $t \notin S$, then $X(t-0)$ exists and $X(t-0)=X(t)$, so that $x_{\nu}$ takes part in the representation of $X\left(r_{t}\right)$ for some rational $r_{t}<t$, which again means that $x_{\nu}$ belongs to the linear space on the right side of (7). Hence,

$$
\overline{\mathscr{L}}\left\{X(t), t \in F_{\varepsilon}\right\} \subseteq \overline{\mathscr{L}}\{X(t), t \in S\},
$$

which proves (7). This completes the proof.

COROLlARY 2.1. If process $X$ is mean square continuous from at least one side at every $t$, then its linear space is separable.

COROLlARY 2.2. If process $X$ is mean square approximately continuous almost everywhere and, for each $t$, functions $R_{t}$ and $g$ are continuous from the same side at $t$, then $X$ has a measurable modification.

This corollary gives a partial solution to the problem formulated in the introductory part of this paper. The other half of the same problem concerned sufficient conditions under which a second-order process can be, outside of a $t$-set of arbitrary small $m$-measure, uniformly approximated (in the mean square sense) by a measurable process. The next result gives conditions for that measurable process to have continuous trajectories, thus providing a connection between mean square properties of a process and properties of its trajectories.

THEOREM 3. Let process $X$ be such that its covariance function satisfies conditions $\left(C_{1}\right)$ and $\left(C_{2}\right)$ from Theorem 2. Then for each $\varepsilon>0$ there are a set $G_{\varepsilon}, m\left(G_{\varepsilon}^{c}\right)<\varepsilon$, and a sequence $\left\{Y_{n}^{\varepsilon}\right\}, Y_{n}^{\varepsilon}=Y_{n}^{\varepsilon}(t), t \in(0 ; 1)$, of measurable second-order processes with continuous trajectories, having the following properties:

(i) Almost everywhere on $G_{\varepsilon}$, process $X$ is a uniform mean square limit of the sequence $\left\{Y_{n}^{\varepsilon}\right\}$,

(ii) $Y_{n}^{\varepsilon}(t) \in H(X)$ for each $n$ and each $t$.

Proof. Let $F_{\varepsilon / 3}$ be a closed set of $m$-measure bigger than $1-\varepsilon / 3$, such that the linear space $\overline{\mathscr{L}}\left\{X(t), t \in F_{\varepsilon / 3}\right\}$ is separable (the existence of such a set is ensured by Theorem 2), and let $\left\{\xi_{n}, n=1,2, \ldots\right\}$ be an orthogonal basis of that linear space. Then process $X_{\varepsilon}$, defined by

$$
X_{\varepsilon}(t)=\sum_{n=1}^{\infty} s_{n}(t) \xi_{n}, \quad s_{n}(t)=E\left[X(t) \xi_{n}\right], t \in(0 ; 1),
$$

is such that

$$
\left\{t: P\left\{X_{\varepsilon}(t) \neq X(t)\right\}>0\right\} \subseteq F_{\varepsilon / 3}^{c} .
$$

Inequality $\left|s_{n}(t)-s_{n}(u)\right| \leqslant E|X(t)-X(u)|^{2} \cdot E\left|\xi_{n}\right|^{2}$ and Corollary 1.1 imply that each function $s_{n}$ is approximately continuous almost everywhere, i.e., that each function $s_{n}$ is measurable. Let $c_{1}, c_{2}, \ldots$ be a decreasing sequence of positive numbers such that $\sum_{n=1}^{\infty} c_{n}=1$. For each $n$ and each $\varepsilon>0$, there is a closed set $E_{n ; \varepsilon / 3}, m\left(E_{n ; \varepsilon / 3}^{c}\right) \leqslant(\varepsilon / 3) c_{n}$, on which function $s_{n}$ is continuous (see [5, Theorem 
21.4]). Thus all functions $s_{1}, s_{2}, \ldots$ are continuous on a set $E_{\varepsilon / 3}=\bigcap_{n=1}^{\infty} E_{n ; \varepsilon / 3}$, which is such that

$$
m\left(E_{\varepsilon / 3}^{c}\right)=m\left(\bigcup_{n=1}^{\infty} E_{n ; \varepsilon / 3}^{c}\right) \leqslant \frac{\varepsilon}{3} .
$$

Obviously, all trajectories of the process $X_{\varepsilon}$ are continuous on $E_{\varepsilon / 3}$. Thus, process $X_{\varepsilon}$ has continuous trajectories on a closed set $F_{\varepsilon / 3} \cap E_{\varepsilon / 3}$ and is a modification of $X$ on that set; it is $m\left(\left(F_{\varepsilon / 3} \cap E_{\varepsilon / 3}\right)^{c}\right) \leqslant 2 \varepsilon / 3$. Let $s_{n}^{*}$ be a function defined on $F_{\varepsilon / 3} \cap E_{\varepsilon / 3}$ by $s_{n}^{*}(t)=I_{F_{\varepsilon / 3} \cap E_{\varepsilon / 3}}(t) s_{n}(t)$, and let $s_{n, c}$ be a continuous extension of $s_{n}^{*}$ on $(0 ; 1)$. Now, if a sequence $\left\{Y_{n}^{\varepsilon}\right\}$ of stochastic processes is defined by

$$
Y_{n}^{\varepsilon}(t)=\sum_{i=1}^{n} s_{i, c}(t) \xi_{i}, \quad t \in(0 ; 1)
$$

then it is easy to see that it has the following properties:

For each $n$, all trajectories of $Y_{n}^{\varepsilon}$ are continuous on $(0 ; 1)$.

For each $t \in F_{\varepsilon / 3} \cap E_{\varepsilon / 3}$, sequence $\left\{Y_{n}^{\varepsilon}(t)\right\}$ converges in the mean square to $X(t)$.

(11) For each $n$, process $Y_{n}^{\varepsilon}$ is mean square continuous and thus measurable.

Statement (ii) follows from (8). If we show that there is a set $G_{\varepsilon} \subseteq F_{\varepsilon / 3} \cap E_{\varepsilon / 3}$, $m\left(F_{\varepsilon / 3} \cap E_{\varepsilon / 3} \cap G_{\varepsilon}^{c}\right) \leqslant \varepsilon / 3$, such that the mean square convergence of sequence $\left\{Y_{n}^{\varepsilon}\right\}$ to $X$ is uniform on $G_{\varepsilon}$, then (because of $m\left(G_{\varepsilon}^{c}\right)=m\left(F_{\varepsilon / 3} \cap E_{\varepsilon / 3} \cap G_{\varepsilon}^{c}\right)+$ $\left.m\left(\left(F_{\varepsilon / 3} \cap E_{\varepsilon / 3}\right)^{c}\right) \leqslant \varepsilon\right)$ this means that (i) holds and the proof will be completed.

Let us consider a measurable process $X_{m}$ such that set $\left\{t: P\left\{X_{m}(t) \neq X(t)\right\}>0\right\}$ has $m$-measure zero (Theorem 2 ). For each $i=1,2, \ldots$ and each $k=1,2, \ldots$ set

$$
\left\{t \in F_{\varepsilon / 3} \cap E_{\varepsilon / 3}:\left\|Y_{i}^{\varepsilon}(t)-X_{m}(t)\right\| \leqslant 1 / k\right\}
$$

is measurable (see e.g., [3, Theorem 21.12]), so that, for any $n$, set

$$
G_{k, n}=\bigcap_{i=n}^{\infty}\left\{t \in F_{\varepsilon / 3} \cap E_{\varepsilon / 3}:\left\|Y_{i}^{\varepsilon}(t)-X_{m}(t)\right\| \leqslant \frac{1}{k}\right\}
$$

is also measurable. For each fixed $k, G_{k, n_{1}} \subseteq G_{k, n_{2}}$ for $n_{1} \leqslant n_{2}$, so that $\lim _{n \rightarrow \infty} m\left(G_{k, n}\right)=m\left(\bigcup_{n=1}^{\infty} G_{k, n}\right)$. If $H \subseteq F_{\varepsilon / 3} \cap E_{\varepsilon / 3}$ is a set on which $X_{m}$ is a mean square limit of sequence $\left\{Y_{n}\right\}$ (because of (10), $H$ might differ from $F_{\varepsilon / 3} \cap E_{\varepsilon / 3}$ only on a set of $m$-measure zero), then, obviously, $H \subseteq \bigcup_{n=1}^{\infty} G_{k, n}$, so that we get

$$
\lim _{n \rightarrow \infty} m\left(G_{k, n}\right) \geqslant m(H)=m\left(F_{\varepsilon / 3} \cap E_{\varepsilon / 3}\right),
$$

and thus (because $\left.\bigcup_{n=1}^{\infty} G_{k, n} \subseteq F_{\varepsilon / 3} \cap E_{\varepsilon / 3}\right) \lim _{n \rightarrow \infty} m\left(G_{k, n}^{c}\right)=0$. This means that, for given $\varepsilon>0$, there is $n_{k}=n_{k}(\varepsilon)$ such that $m\left(F_{\varepsilon / 3} \cap E_{\varepsilon / 3} \cap G_{k, n_{k}}^{c}\right) \leqslant \varepsilon /\left(3 \cdot 2^{k}\right)$. Set $G_{\varepsilon}=\bigcap_{k=1}^{\infty} G_{k, n_{k}}$ will be measurable and its measure will be

$$
m\left(G_{\varepsilon}\right) \geqslant 1-2 \varepsilon / 3-m\left(F_{\varepsilon / 3} \cap E_{\varepsilon / 3} \cap G_{\varepsilon}^{c}\right) \geqslant 1-\varepsilon .
$$

Beside that, we will have $\left\|Y_{i}^{\varepsilon}(t)-X_{m}(t)\right\| \leqslant 1 / k$ for each $i \geqslant n_{k}$ and $t \in G_{k, n_{k}}$; thus (because $G_{\varepsilon} \subseteq G_{k, n_{k}}$ ) inequality $\left\|Y_{i}^{\varepsilon}(t)-X_{m}(t)\right\| \leqslant 1 / k$ will be satisfied for 
each $i \geqslant n_{k}$ and each $t \in G_{\varepsilon}$, as we wanted to prove. This means that on a set $G_{\varepsilon}$, $m\left(G_{\varepsilon}\right) \geqslant 1-\varepsilon$, process $X_{m}$ is a mean square uniform limit of a sequence $\left\{Y_{n}^{\varepsilon}\right\}$, and thus a mean square convergence of $\left\{Y_{n}^{\varepsilon}\right\}$ to $X$ is uniform almost everywhere on $G_{\varepsilon}$. The proof is completed.

\section{REFERENCES}

1. S. Cambanis, The measurability of a stochastic process of second order and its linear space, Proc. Amer. Math. Soc. 17 (1975), 467-475.

2. R. O. Davies, Separate approximate continuity implies measurability, Proc. Cambridge Philos. Soc. 73 (1973), 461-465.

3. E. Hewitt and K. Stromberg, Real and abstract analysis, Springer-Verlag, Berlin, 1965.

4. E. W. Hobson, The theory of functions of a real variable, Vol. 1, Warren Press, Washington, D. C., 1950.

5. M. E. Munroe, Introduction to measure and integration, Addison-Wesley, Reading, Mass., 1959.

Department of Statistics and Probability, Michigan State University, East Lansing, MICHIGAN 48824 